\title{
Triangle Singularity as the Origin of the $a_{1}(1420)$
}

G. D. Alexeev, ${ }^{7}$ M. G. Alexeev, ${ }^{25,26}$ A. Amoroso, ${ }^{25,26}$ V. Andrieux, ${ }^{9,28}$ V. Anosov, ${ }^{7}$ A. Antoshkin, ${ }^{7}$ K. Augsten, ${ }^{7,18}$ W. Augustyniak, ${ }^{29}$ C. D. R. Azevedo, ${ }^{1}$ B. Badełek,${ }^{30}$ F. Balestra, ${ }^{25,26}$ M. Ball, ${ }^{3}$ J. Barth, ${ }^{3}$ R. Beck, ${ }^{3}$ Y. Bedfer,${ }^{20}$ J. Berenguer Antequera, ${ }^{25,26}$ J. Bernhard, ${ }^{12,9}$ M. Bodlak, ${ }^{17}$ F. Bradamante, ${ }^{24}$ A. Bressan, ${ }^{23,24}$ V. E. Burtsev, ${ }^{27}$ W.-C. Chang, ${ }^{21}$ C. Chatterjee, ${ }^{23,24}$ M. Chiosso, ${ }^{25,26}$ A. G. Chumakov, ${ }^{27}$ S.-U. Chung, ${ }^{15, b}$ A. Cicuttin, ${ }^{24, c}$ P. M. M. Correia, ${ }^{1}$ M. L. Crespo, ${ }^{24, c}$ D. D’Ago, ${ }^{23,24}$ S. Dalla Torre, ${ }^{24}$ S. S. Dasgupta, ${ }^{6}$ S. Dasgupta, ${ }^{24}$ I. Denisenko, ${ }^{7}$ O. Yu. Denisov,${ }^{26}$ S. V. Donskov, ${ }^{19}$ N. Doshita, ${ }^{32}$ Ch. Dreisbach, ${ }^{15}$ W. Dünnweber, ${ }^{\mathrm{n}}$ R. R. Dusaev, ${ }^{27}$ A. Efremov, ${ }^{7, a}$ P. D. Eversheim, ${ }^{3}$ P. Faccioli, ${ }^{11}$ M. Faessler, ${ }^{n}$ M. Finger, ${ }^{17}$ M. Finger, Jr., ${ }^{17}$ H. Fischer, ${ }^{8}$ C. Franco, ${ }^{11}$ J. M. Friedrich, ${ }^{15}$ V. Frolov, ${ }^{7,9}$ F. Gautheron, ${ }^{2,28}$ O. P. Gavrichtchouk, ${ }^{7}$ S. Gerassimov, ${ }^{14,15}$ J. Giarra, ${ }^{12}$ I. Gnesi, ${ }^{25,26}$ M. Gorzellik, ${ }^{8}$ A. Grasso, ${ }^{25,26}$ A. Gridin, ${ }^{7}$

M. Grosse Perdekamp, ${ }^{28}$ B. Grube,${ }^{15}$ A. Guskov, ${ }^{7}$ D. von Harrach, ${ }^{12}$ R. Heitz, ${ }^{28}$ F. Herrmann, ${ }^{8}$ N. Horikawa, ${ }^{16, d}$ N. d'Hose, ${ }^{20}$ C.-Y. Hsieh, ${ }^{21, \mathrm{e}}$ S. Huber, ${ }^{15}$ S. Ishimoto, ${ }^{32, \mathrm{f}}$ A. Ivanov, ${ }^{7}$ T. Iwata ${ }^{32}$ M. Jandek, ${ }^{18}$ V. Jary, ${ }^{18}$ R. Joosten, ${ }^{3}$ P. Jörg, ${ }^{8,}$ ${ }^{g}$ E. Kabuß, ${ }^{12}$ F. Kaspar, ${ }^{15}$ A. Kerbizi, ${ }^{23,24}$ B. Ketzer, ${ }^{3}$ G. V. Khaustov, ${ }^{19}$ Yu. A. Khokhlov, ${ }^{19, h}$ Yu. Kisselev, ${ }^{7, a}$ F. Klein, ${ }^{4}$ J. H. Koivuniemi, ${ }^{2,28}$ V. N. Kolosov, ${ }^{19}$ K. Kondo Horikawa, ${ }^{32}$ I. Konorov, ${ }^{14,15}$ V. F. Konstantinov, ${ }^{19}$ A. M. Kotzinian, ${ }^{26, i}$ O. M. Kouznetsov, ${ }^{7}$ A. Koval, ${ }^{29}$ Z. Kral,${ }^{17}$ F. Krinner, ${ }^{15}$ Y. Kulinich, ${ }^{28}$ F. Kunne, ${ }^{20}$ K. Kurek, ${ }^{29}$ R. P. Kurjata, ${ }^{31}$ A. Kveton, ${ }^{17}$ K. Lavickova, ${ }^{17}$ S. Levorato, ${ }^{24,9}$ Y.-S. Lian, ${ }^{21, j}$ J. Lichtenstadt, ${ }^{22}$ P.-J. Lin, ${ }^{20}$ R. Longo, ${ }^{28}$ V. E. Lyubovitskij,,${ }^{27, k}$ A. Maggiora, ${ }^{26}$ A. Magnon, ${ }^{6}$ N. Makins,${ }^{28}$ N. Makke, ${ }^{24, c}$ G. K. Mallot, ${ }^{9,8}$ A. Maltsev, ${ }^{7}$ S. A. Mamon, ${ }^{27}$ B. Marianski, ${ }^{29, a}$ A. Martin, ${ }^{23,24}$ J. Marzec, ${ }^{31}$ J. Matoušek, ${ }^{23,24}$ T. Matsuda, ${ }^{13}$ G. Mattson, ${ }^{28}$ G. V. Meshcheryakov, ${ }^{7}$ M. Meyer, ${ }^{28,20}$ W. Meyer, ${ }^{2}$ Yu. V. Mikhailov, ${ }^{19}$ M. Mikhasenko, ${ }^{3,9}$ E. Mitrofanov, ${ }^{7}$ N. Mitrofanov, ${ }^{7}$ Y. Miyachi, ${ }^{32}$ A. Moretti, ${ }^{23,24}$ A. Nagaytsev, ${ }^{7}$ C. Naim, ${ }^{20}$ D. Neyret,${ }^{20}$ J. Nový, ${ }^{18}$ W.-D. Nowak, ${ }^{12}$ G. Nukazuka, ${ }^{32}$ A. S. Nunes, ${ }^{11,1}$ A. G. Olshevsky, ${ }^{7}$ M. Ostrick, ${ }^{12}$ D. Panzieri, ${ }^{26, \mathrm{~m}}$ B. Parsamyan, ${ }^{25,26}$ S. Paul,${ }^{15}$ H. Pekeler, ${ }^{3}$ J.-C. Peng, ${ }^{28}$ M. Pešek, ${ }^{17}$ D. V. Peshekhonov, ${ }^{7}$ M. Pešková, ${ }^{17}$ N. Pierre, ${ }^{12,20}$ S. Platchkov, ${ }^{20}$ J. Pochodzalla, ${ }^{12}$ V. A. Polyakov, ${ }^{19}$ J. Pretz, ${ }^{4,0}$ M. Quaresma, ${ }^{21,11}$ C. Quintans, ${ }^{11}$ G. Reicherz, ${ }^{2}$ C. Riedl, ${ }^{28}$ T. Rudnicki, ${ }^{30}$ D. I. Ryabchikov, ${ }^{19,15}$ A. Rybnikov, ${ }^{7}$ A. Rychter, ${ }^{31}$ V. D. Samoylenko, ${ }^{19}$ A. Sandacz, ${ }^{29}$ S. Sarkar, ${ }^{6}$ I. A. Savin, ${ }^{7}$ G. Sbrizzai, ${ }^{23,24}$ H. Schmieden, ${ }^{4}$ A. Selyunin, ${ }^{7}$ L. Sinha, ${ }^{6}$ M. Slunecka, ${ }^{17}$ J. Smolik, ${ }^{7}$ A. Srnka, ${ }^{5}$ D. Steffen, ${ }^{9,15}$ M. Stolarski, ${ }^{11}$ O. Subrt, ${ }^{9,18}$ M. Sulc, ${ }^{10}$ H. Suzuki, ${ }^{32, d}$ P. Sznajder, ${ }^{29}$ S. Tessaro,${ }^{24}$ F. Tessarotto $\odot,{ }^{24,9}$ A. Thiel, ${ }^{3}$ J. Tomsa, ${ }^{17}$ F. Tosello, ${ }^{26}$ A. Townsend, ${ }^{28}$ V. Tskhay, ${ }^{14}$ S. Uhl, ${ }^{15}$ B. I. Vasilishin, ${ }^{27}$ A. Vauth, ${ }^{4,9, p}$ B. M. Veit,,${ }^{12,9}$ J. Veloso, ${ }^{1}$ B. Ventura, ${ }^{20}$ A. Vidon, ${ }^{20}$ M. Virius, ${ }^{18}$ M. Wagner, ${ }^{3}$ S. Wallner, ${ }^{15}$ K. Zaremba,${ }^{31}$ P. Zavada, ${ }^{7}$ M. Zavertyaev, ${ }^{14}$ M. Zemko, ${ }^{17,9}$ E. Zemlyanichkina, ${ }^{7}$ Y. Zhao, ${ }^{24}$ and M. Ziembicki ${ }^{31}$

\footnotetext{
${ }^{1}$ Department of Physics, University of Aveiro, I3N, 3810-193 Aveiro, Portugal

${ }^{2}$ Institut für Experimentalphysik, Universität Bochum, 44780 Bochum, Germany

${ }^{3}$ Helmholtz-Institut für Strahlen- und Kernphysik, Universität Bonn, 53115 Bonn, Germany

${ }^{4}$ Physikalisches Institut, Universität Bonn, 53115 Bonn, Germany

${ }^{5}$ Institute of Scientific Instruments of the CAS, 61264 Brno, Czech Republic

${ }^{6}$ Matrivani Institute of Experimental Research \& Education, Calcutta-700 030, India

${ }^{7}$ Joint Institute for Nuclear Research, 141980 Dubna, Moscow region, Russia

${ }^{8}$ Physikalisches Institut, Universität Freiburg, 79104 Freiburg, Germany

${ }^{9}$ CERN, 1211 Geneva 23, Switzerland

${ }^{10}$ Technical University in Liberec, 46117 Liberec, Czech Republic

${ }^{11}$ LIP, 1649-003 Lisbon, Portugal

${ }^{12}$ Institut für Kernphysik, Universität Mainz, 55099 Mainz, Germany

${ }^{13}$ University of Miyazaki, Miyazaki 889-2192, Japan

${ }^{14}$ Lebedev Physical Institute, 119991 Moscow, Russia

${ }^{15}$ Physik Department, Technische Universität München, 85748 Garching, Germany

${ }^{16}$ Nagoya University, 464 Nagoya, Japan

${ }^{17}$ Faculty of Mathematics and Physics, Charles University, 18000 Prague, Czech Republic

${ }^{18}$ Czech Technical University in Prague, 16636 Prague, Czech Republic

${ }^{19}$ State Scientific Center Institute for High Energy Physics of National Research Center "Kurchatov Institute," 142281 Protvino, Russia

${ }^{20}$ IRFU, CEA, Université Paris-Saclay, 91191 Gif-sur-Yvette, France

${ }^{21}$ Academia Sinica, Institute of Physics, Taipei 11529, Taiwan

${ }^{22}$ School of Physics and Astronomy, Tel Aviv University, 69978 Tel Aviv, Israel

${ }^{23}$ Department of Physics, University of Trieste, 34127 Trieste, Italy

${ }^{24}$ Trieste Section of INFN, 34127 Trieste, Italy
} 


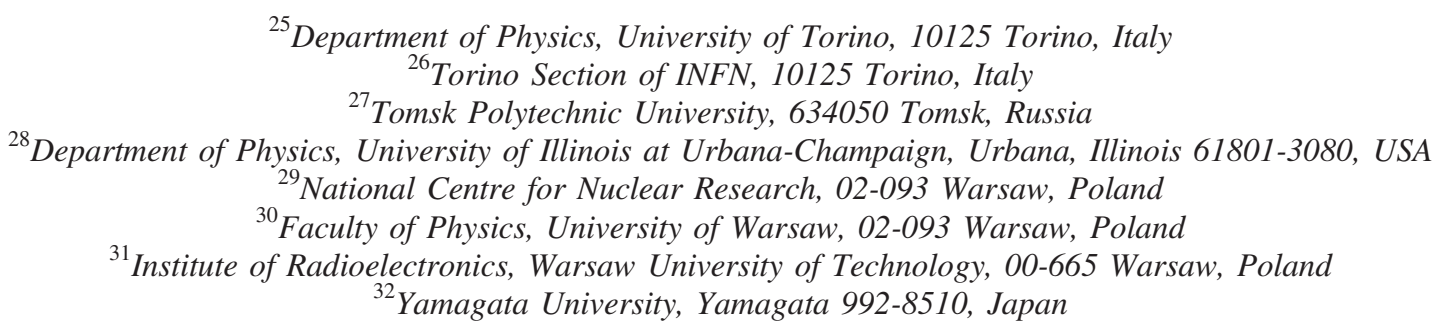

(Received 3 July 2020; revised 4 May 2021; accepted 26 May 2021; published 18 August 2021)

\begin{abstract}
The COMPASS Collaboration experiment recently discovered a new isovector resonancelike signal with axial-vector quantum numbers, the $a_{1}(1420)$, decaying to $f_{0}(980) \pi$. With a mass too close to and a width smaller than the axial-vector ground state $a_{1}(1260)$, it was immediately interpreted as a new light exotic meson, similar to the $X, Y, Z$ states in the hidden-charm sector. We show that a resonancelike signal fully matching the experimental data is produced by the decay of the $a_{1}(1260)$ resonance into $K^{*}(\rightarrow K \pi) \bar{K}$ and subsequent rescattering through a triangle singularity into the coupled $f_{0}(980) \pi$ channel. The amplitude for this process is calculated using a new approach based on dispersion relations. The triangle-singularity model is fitted to the partial-wave data of the COMPASS experiment. Despite having fewer parameters, this fit shows a slightly better quality than the one using a resonance hypothesis and thus eliminates the need for an additional resonance in order to describe the data. We thereby demonstrate for the first time in the lightmeson sector that a resonancelike structure in the experimental data can be described by rescattering through a triangle singularity, providing evidence for a genuine three-body effect.
\end{abstract}

DOI: 10.1103/PhysRevLett.127.082501

Quantum chromodynamics is generally accepted as the fundamental quantum-field theory of the strong interaction. How exactly the spectrum of bound states (hadrons) emerges from the underlying interaction between quarks and gluons is, however, not yet understood. The main difficulty is the rise of the strong coupling at the low-energy scale relevant for hadrons, which makes the theory unsolvable with perturbative methods. Although the constituentquark model [1-3] describes many of the observed mesons, it seems that the spectrum is notably richer: there is growing experimental evidence for bound states beyond the constituent-quark model. Such states are commonly called exotic [4-9]. In addition to mapping out the full spectrum predicted by models and, more recently, by lattice gauge theory [10], the search for such exotic states drives the current interest in hadron spectroscopy.

The study of single-diffractive reactions with a highenergy meson beam, as performed by the COMPASS experiment at the CERN SPS [11,12], is a natural way to investigate meson excitations (for a recent review, see Ref. [13]). In such reactions, at high energies commonly described by the exchange of a Pomeron $\mathbb{P}$, the incoming beam particle is excited by the strong interaction with a proton target. Regge theory then allows us to factorize off

Published by the American Physical Society under the terms of the Creative Commons Attribution 4.0 International license. Further distribution of this work must maintain attribution to the author(s) and the published article's title, journal citation, and DOI. Funded by SCOAP. the target vertex, such that we only consider the beam vertex. Although the produced excited system immediately decays, the reaction products unveil the properties of the excitation. An unprecedented amount of data comprising almost $50 \times 10^{6}$ events for the reaction $\pi^{-}+p \rightarrow$ $\pi^{-} \pi^{-} \pi^{+}+p$ were used by COMPASS to perform a detailed analysis of $\pi_{J}$ and $a_{J}$ mesons with isospin $I=1$, negative $G$ parity, and positive $C$ parity implied by $G=C(-1)^{I}$. The partial-wave analysis (PWA) technique in connection with the isobar model was used to separate excitations with different quantum numbers; see Refs. [13,14] for details. Individual waves are labeled $J^{P C} M^{\epsilon} \xi \pi L$, where $J$ is the total angular momentum of the 3-pion system, $P$ the spatial and $C$ the chargeconjugation parity. The quantum number $M$ labels the projection of the spin $J$ onto the direction of the beam in the rest frame of $\pi^{-} \pi^{-} \pi^{+}$, and $\epsilon$ indicates the reflection symmetry with respect to the production plane. At the high center-of-momentum energies of the experiment, the reflectivity quantum number $\epsilon$ corresponds to the naturality of the exchanged particle and is hence always positive for Pomeron exchange. The orbital angular momentum between the neutral system of two pions (isobar) and the remaining pion is denoted by $L$. The symbol $\xi$ labels the assumed isobar, i.e., the interaction amplitude in the neutral $\pi \pi$ subchannel.

A PWA including 88 waves in total was performed separately in 100 bins of the $3 \pi$ invariant mass $m_{3 \pi}$ and 11 bins of the reduced 4-momentum transfer squared $t^{\prime}$ [see Eq. (6) in Ref. [14]]. The results are summarized in 

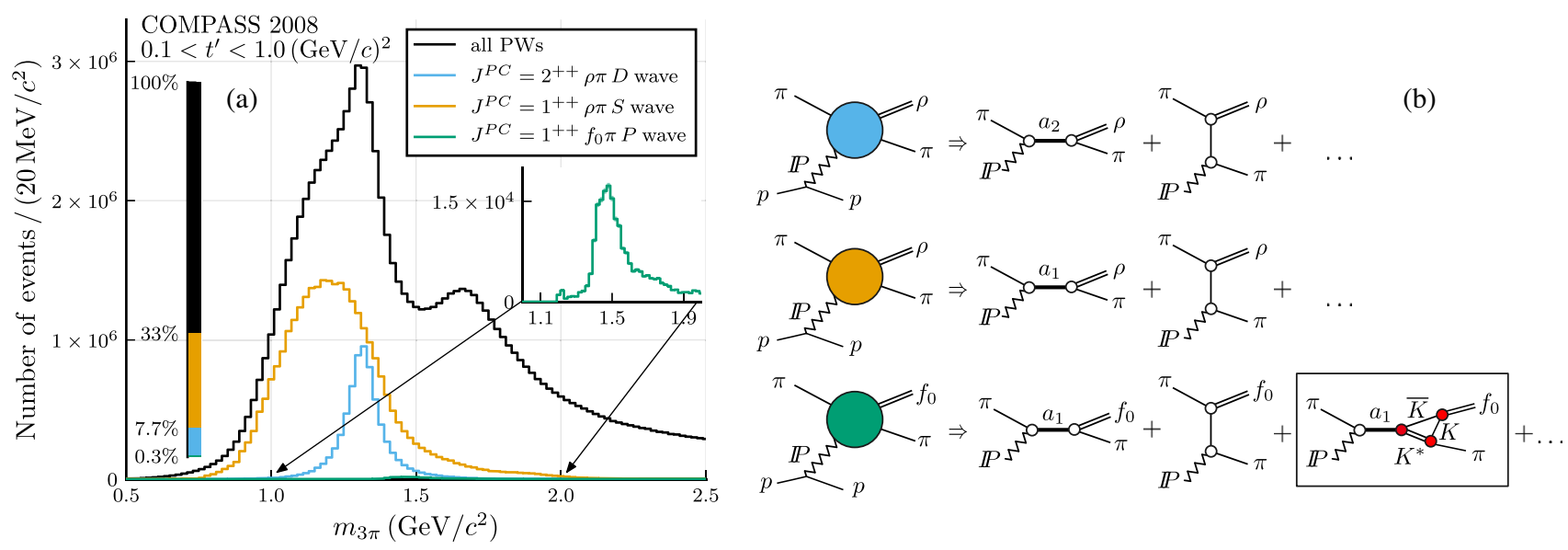

FIG. 1. (a) Intensities of selected waves from the PWA of the reaction $\pi^{-}+p \rightarrow \pi^{-} \pi^{-} \pi^{+}+p$ [14]. The inset shows an enlarged view of the $1^{++} 0^{+} f_{0} \pi P$ wave. The colored bar on the left indicates the contributions of the different waves to the total intensity. (b) Diagrams showing possible contributions to the $\rho(770) \pi$ and $f_{0}(980) \pi$ production amplitudes. The Pomeron is labeled $\mathbb{P}, a_{1}$ refers to the axialvector ground state $a_{1}(1260)$, and $a_{2}$ to the tensor ground state $a_{2}(1320)$. The framed diagram shows the dominant contribution to the $a_{1}(1420)$ signal via the triangle diagram.

Fig. 1(a), where we show the intensities of selected waves as a function of $m_{3 \pi}$, summed over all $t^{\prime}$ bins. Among many important observations, an exotic resonancelike signal with quantum numbers $J^{P C}=1^{++}$was found in the $1^{++} 0^{+} f_{0} \pi P$ wave as a clear peak at $1.4 \mathrm{GeV} / c^{2}[15]$ [see inset of Fig. 1(a)]. The resonancelike behavior was corroborated by the observed phase motion, i.e., a massdependent relative phase with respect to several other reference waves. Extensive studies, also using the "freed-isobar" method [16], undoubtedly confirmed the signal and proved that it was not an artifact of any particular isobar parametrization [14]. Following the Particle Data Group (PDG) convention, the signal was called $a_{1}(1420)$ according to its quantum numbers $I^{G}\left(J^{P C}\right)=1^{-}\left(1^{++}\right)$. It was immediately realized that it could not be an ordinary quark-model meson resonance: (i) with about $150 \mathrm{MeV} / c^{2}$, its width is much smaller than that of the axial-vector ground state $a_{1}(1260)$ of about $500 \mathrm{MeV} / c^{2}$; (ii) the signal is separated from the ground state by only about $150 \mathrm{MeV} / c^{2}$, whereas the energy difference between different radial excitation levels is typically $400 \mathrm{MeV} / c^{2}$ as estimated based on the slope of the radial excitation trajectory $[17,18]$; (iii) so far, the $a_{1}(1420)$ was seen only in the $f_{0}(980) \pi$ final state.

Various interpretations followed the $a_{1}(1420)$ observation [19-23], requiring or not a new resonance. Resonances are consistently introduced in general scattering theory [24], where the reaction amplitude is an analytic function of the total energy squared $s$ that is regarded as a complex number; they are found as poles on the unphysical sheet of the complex $s$ plane attached to the real axis from below. In explanations involving either diquark-antidiquark molecules or tightly bound tetraquarks, the observed signal, i.e., peak and phase motion, is caused by a pole-type singularity located on the closest sheet. Alternatively, a so-called triangle-singularity (TS) mechanism [24-26] was proposed as the mechanism behind the $a_{1}(1420)$ signal [19]. Here, a logarithmic branch point caused by a coupledchannel effect, particularly by the $K^{*} \bar{K}-f_{0} \pi$ interaction, is located near the physical region on the closest unphysical sheet. The other proposed model [23,27] that does not require a new resonance pole combines resonant and nonresonant production mechanisms resulting in a peak in the $1^{++} 0^{+} f_{0} \pi P$ wave. However, the generated phase motion is at the position of the $a_{1}(1260)$ resonance, which is inconsistent with observation.

In this Letter, we interpret the COMPASS data in terms of the triangle-singularity model based on a new method for the calculation of the amplitude. The calculation implements the proposal of Ref. [28] exploiting the unitarity and analyticity properties of the amplitude. The new model goes beyond Ref. [19] by incorporating spin in a more systematic way and allowing us to address higher-order rescattering effects. To our knowledge, this is the first time that the TS model, mimicking a resonance signal, is fitted successfully to experimental data in the light-meson sector describing both intensity and phase motion simultaneously. Comparable studies in the heavy-quark sector, see, e.g., Refs. $[7,29,30]$, were performed on a much smaller statistical basis.

The dynamics of a hadronic three-body system is commonly understood in terms of quasi-two-body interactions with subchannel resonances $\xi$ decaying further into pairs of final-state particles. Often, however, the same final state can be obtained through several decay chains when the two-particle interaction is non-negligible for different particle pairs [31,32]. Different decay chains are coherent; hence they interfere. The unitarity of the scattering matrix enforces a consistency relation between the different chains [33-35]. This relation makes the line shapes of 
the resonances in a particle pair in a system of three particles dependent on the dynamics in the other pairs [3639]. An equivalent way of describing this interrelation between pairwise interactions is to state that the crosschannel two-body resonances in the $\pi \pi, K \pi$, and $\bar{K} K$ systems rescatter to one another, thereby modifying the original undistorted line shapes. In addition, the probabilities for a three-body resonance decaying to one or another channel may be redistributed due to final-state interaction [40,41]. The latter effect is strongly enhanced for certain kinematic conditions [24,42] and produces the observed resonancelike signal in the case considered here.

We find that the presence of the $K^{*}(892)$ resonance (hereafter referred to as $K^{*}$ ) in the $K \bar{K} \pi$ channel drastically affects the $f_{0}(980) \pi$ channel, since the rescattering between $K^{*} \rightarrow K \pi P$ wave and $K \bar{K} \rightarrow f_{0}(980) \rightarrow \pi \pi S$ wave occurs with all intermediate particles being almost on their mass shell for $m_{3 \pi} \approx 1.4 \mathrm{GeV} / c^{2}$, i.e., slightly above the $K^{*} \bar{K}$ threshold [19]. This effect does not disturb the narrow line shape of the $f_{0}(980)$, but it leads to a significant redistribution of the $a_{1}(1260)$ decay probabilities. The originally negligible $f_{0}(980) \pi P$-wave decay channel is populated by the rescattering from the $K^{*} \bar{K}$ decay locally around $1.4 \mathrm{GeV} / c^{2}$.

Our calculation of the TS amplitude is reminiscent of the Khuri-Treiman (KT) equation first developed in 1960 [3335]: the dispersion relation and two-body unitarity are used to connect the isobar amplitude with the partial-wave projection of the cross channels. By $F_{W}^{\{1\}}$ we denote the production amplitude of a three-particle system (123) with a given set of quantum numbers $W$, the invariant mass squared $s \equiv m_{3 \pi}^{2}$, and the isobar formed by particles 2 and 3 (hereafter labeled $\{1\}$ using indices in curly brackets) with invariant mass squared $\sigma_{\{1\}} \equiv\left(p_{2}+p_{3}\right)^{2}$. We write the dispersion relation for the kinematic-singularity-free amplitude $F_{W}^{\{1\}}\left(s, \sigma_{\{1\}}\right) / K_{W}^{\{1\}}\left(s, \sigma_{\{1\}}\right)$, with $K_{W}^{\{1\}}$ being the breakup momentum for the $f_{0} \pi$ system required for the $P$-wave, see Supplemental Material [43]:

$$
\begin{aligned}
F_{W}^{\{1\}}\left(s, \sigma_{\{1\}}\right)= & K_{W}^{\{1\}}\left(s, \sigma_{\{1\}}\right)\left(C_{W}^{\{1\}}(s)\right. \\
& \left.+\frac{1}{2 \pi} \sum_{\omega} \int_{\sigma_{\mathrm{th}, W}}^{\infty} \frac{\rho_{\omega}(\sigma) \hat{F}_{W, \omega}^{\{1\}}(s, \sigma)}{K_{W}^{\{1\}}(s, \sigma)\left(\sigma-\sigma_{\{1\}}-i \varepsilon\right)} d \sigma\right) .
\end{aligned}
$$

Here, the indices $W$ and $\omega$ refer to the full set of quantum numbers labeling a given wave. For the $f_{0} \pi P$ wave considered in this Letter, the term $C_{W}^{\{1\}}(s)$ parametrizes the three-body production dynamics and the decay into the given final state $W$. It includes the direct production of the $a_{1}(1260)$ resonance and a term for the nonresonant production that is further described below. The sum runs over all possible cross channels with quantum numbers $\omega$.
In the dispersion integral, $\rho_{\omega}(\sigma)$ is the two-body phasespace factor, and $\hat{F}_{W, \omega}^{\{1\}}(s, \sigma)$ is the projection of the cross channel $\{3\}$, i.e., the isobar formed by particles 1 and 2 , with quantum numbers $\omega$ onto channel $\{1\}$ with quantum numbers $W$. We do not expect isobars in channel $\{2\}$, formed by, e.g., $K^{-} \pi^{-}, K^{0} \pi^{-}$, or $\pi^{-} \pi^{-}$. We note an important difference to the original KT equation. The latter constrains the subchannel dynamics in the three-body system. The total invariant mass of the system is treated as a fixed parameter in the model. In 1965, Aitchison suggested that this parametric dependence is actually physical and represents the three-body interaction [32]. In Ref. [28], the authors demonstrated that the KT kernel can be used to separate the genuine three-body dynamics from the final-state interaction. Correspondingly, in Eq. (1) the direct decay of $a_{1}(1260)$ enters in $C_{W}^{\{1\}}(s)$, while the $s$ dependent dispersion integral adds the rescattering corrections. Assuming that modifications of the line shapes of the cross-channel resonances due to rescattering are negligible, we find that the $K^{*} \bar{K}$ channel produces a narrow peak and a strong phase motion at the mass of the $a_{1}(1420)$ due to the TS being very close to the physical region, while all other possible rescattering corrections, which we investigated, manifest themselves in a broad bump and a slow phase motion similar to the direct decay and the nonresonant background (see Supplemental Material [43]).

For a fit of the TS model to the COMPASS spin-density matrix elements [48], referred to below as the data points, we choose the three waves depicted in Fig. 1(b), which constitute the dominant contributions to the $\rho \pi$ and $f_{0} \pi$ production amplitudes: (i) the $1^{++} 0^{+} \rho \pi S$ wave describes the source of the rescattering process, since its largest contribution comes from the $a_{1}(1260)$. This wave also contains a significant contribution from nonresonant "Deck-like" processes [49]; (ii) the $1^{++} 0^{+} f_{0} \pi P$ wave contains the $a_{1}(1420)$ signal; (iii) the $2^{++} 1^{+} \rho \pi D$ wave exhibits a clean $a_{2}(1320)$ resonance and is included in order to fix the relative phases and stabilize the fit. In general, there are two components for each wave in the model: a resonance amplitude, i.e., a propagator that contains a pole [in this case either the $a_{1}(1260)$ or the $\left.a_{2}(1320)\right]$, and a component with $t$-channel $\pi$ exchange accounting for nonresonant processes. We parametrize the $a_{1}(1260)$ propagator by a relativistic Breit-Wigner (BW) amplitude with energy-dependent width saturated by the $\rho \pi$ decay channel [50]. For the resonance part of the $\rho \pi D$ wave we employ the $a_{2}(1320)$ propagator parametrized by a BW amplitude with dynamical width including the $\rho \pi(80 \%)$ and $\eta \pi(20 \%)$ channels, as discussed in Ref. [50]. The nonresonant background is added coherently to each wave. We use an empirical parametrization given by $\left(m_{3 \pi} / m_{0}-1\right)^{b} \exp \left[-\left(c_{0}+c_{1} t^{\prime}+c_{2} t^{\prime 2}\right) \tilde{p}^{2}\right]$, where $\tilde{p}$ is an effective breakup momentum for the decay into $\xi \pi$ at the given $m_{3 \pi}$ value, taking into account the finite width of the isobar $\xi$ and the Bose symmetry of the system, and 
$m_{0}=0.5 \mathrm{GeV} / c^{2}$ [see Eqs. (27) and (29) in Ref. [50] ]. For the model calculations, the $t^{\prime}$ value is fixed to the lower edge of the respective bin. For the $f_{0} \pi P$ wave, the resonance part of the production amplitude is modified by the $K^{*} \bar{K} \rightarrow f_{0} \pi$ rescattering via the TS. As the direct decay of the $a_{1}(1260)$ to the $f_{0} \pi$ final state has a very slow phase motion and a similar shape as the phenomenological parametrization of the nonresonant part due to the limited phase space (see Supplemental Material [43] for details), the fit cannot distinguish between the two components. Therefore, this additional component is only considered for systematic studies.

The free parameters of the model, i.e., the $t^{\prime}$-dependent complex couplings, the background parameters $b$ and $c_{i}$, as well as the $t^{\prime}$-independent BW parameters, are determined by a fit to the COMPASS data points in $m_{3 \pi}$ and $t^{\prime}$ bins. We note that there are no free parameters influencing the line shape of the TS amplitude, while the strength and the background parameters are adjusted in the fit. As explained in more detail in Ref. [50], the data points $y_{i}$ to be fitted are the intensity and the real and imaginary parts of the interference terms for the 3 selected waves inside the chosen $m_{3 \pi}$ ranges (indicated in Fig. 2) for all $11 t^{\prime}$ bins. The fit is performed by minimizing the sum of the squared differences between data points $y_{i}$ and model prediction $\hat{y}_{i}$, weighted by the inverse squared statistical uncertainties:

$$
\mathcal{R}^{2}=\sum_{i} \frac{\left(y_{i}-\hat{y}_{i}\right)^{2}}{\sigma_{i}^{2}}
$$

Figure 2 shows the result of the TS model fit in the lowest $t^{\prime}$ bin, selecting only the $f_{0} \pi P$ wave (full lines). The fit results for all three waves in all $11 t^{\prime}$ bins can be found in the Supplemental Material [43]. Figure 2(a) shows the intensity of the $f_{0} \pi P$ wave and Fig. 2(b) the relative phase to the $\rho \pi S$ wave, both as a function of $m_{3 \pi}$. The resonancelike behavior of the TS amplitude is most evident from the circle in the Argand diagram in Fig. 2(c). The nonresonant background (green arrows) helps to slightly adjust the position of the circle. Since the phase of the background component does not change with $m_{3 \pi}$, all green arrows are parallel.

In order to evaluate the quality of the TS model fit, we also perform a fit to the data using a simple BW description of the $a_{1}(1420)$ signal instead of the TS amplitude. This is accomplished by replacing the TS parametrization of the $f_{0} \pi P$ wave by a relativistic BW amplitude with free mass and width parameters assuming the $a_{1}(1420)$ being a genuine new resonance. We use a constant-width parametrization since further decay modes of this hypothetical new particle are unknown. Figure 2 shows that the fits with the BW model (dashed lines) and the TS model (solid lines) are of very similar quality. Both models are capable of describing the intensities as well as the corresponding interference terms. For a quantitative comparison, one can use the quantity defined in Eq. (2). The biggest contribution comes from the $\rho \pi S$ and $\rho \pi D$ waves. Since the description of these two waves is very similar in both fit models, we can omit them for the comparison of the fit quality. In addition, we can exclude one of the two remaining phases of the interferences, since they depend linearly on one another. Defining $\mathcal{R}_{\text {red }}^{2}$ as the reduced weighted sum of the remaining residuals squared divided by the number of degrees of freedom, where only the fit parameters specific to the $f_{0} \pi P$ wave are taken into account, we arrive at a value of $\mathcal{R}_{\text {red,TS }}^{2}=4.8$ for the TS and $\mathcal{R}_{\text {red,BW }}^{2}=5.2$ for the $\mathrm{BW}$
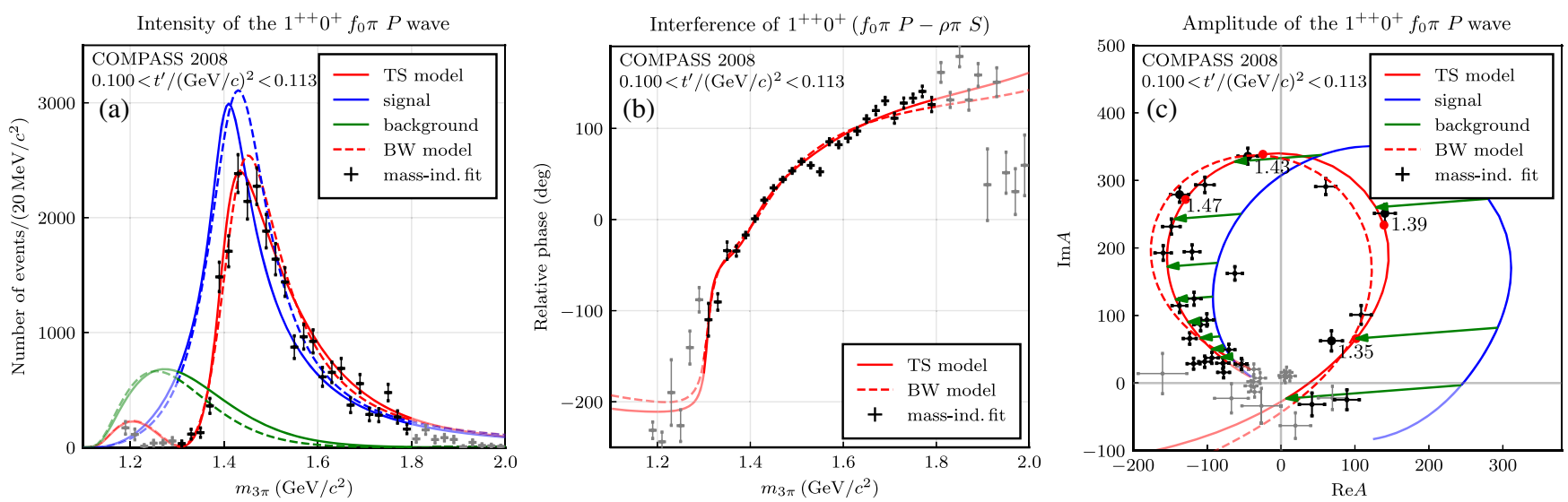

FIG. 2. Results of the fit with the TS model (solid lines) and the BW model (dashed lines) to the wave with the $a_{1}(1420)$ resonancelike signal. The fit range is indicated by the color saturation of data points and lines. (a) Intensity of the $1^{++} 0^{+} f_{0} \pi P$ wave. The complete fit model (red) is decomposed into its signal (blue) and background (green) contributions. (b) Relative phase between the $1^{++} 0^{+} \rho \pi S$ wave and the $1^{++} 0^{+} f_{0} \pi P$ wave. (c) Argand diagram. The red dots on the TS-model curve correspond to the indicated $m_{3 \pi}$ values in units of $\mathrm{GeV} / c^{2}$. 
model. The values indicate that the two fits have comparable quality. The advantage of the TS model is that it has two fit parameters less, since it does not require a new particle with corresponding mass and width.

To study the stability of the result, we investigate a wide range of sources of systematic uncertainties, both with respect to changes of the model and to changes of the data points. We perform fits where the data points $y_{i}$ are varied according to systematic studies for the PWA in bins of mass and $t^{\prime}$, published in Ref. [14]. These include using a smaller wave set, removing negative reflectivity waves, relaxing the event selection, using a model with relaxed coherence assumption (see Ref. [14] for details), or changing the parametrization of the $f_{0}(980)$. In an additional study, we use the result of a statistical reanalysis of Ref. [14] applying the bootstrap technique [51]. Also, we consider several variations in the fit model for the TS: (i) a fit with nonBose-symmetrized phase space; (ii) neglecting the spins of the particles involved (similar to Ref. [19]); (iii) including the excitations $a_{1}(1640)$ and $a_{2}(1700)$ in the $\rho \pi S$ and $\rho \pi D$ waves, respectively (mass and width fixed to the values from the PDG [1]); and (iv) varying mass and width of the $K^{*}$ resonance according to their uncertainties [1] in order to estimate the effect of further rescattering. The TS model systematically yields a slightly smaller $\mathcal{R}_{\text {red }}^{2}$ than the BW model; see Supplemental Material [43].

In summary, we have shown that the recently discovered resonancelike signal $a_{1}(1420)$ can be fully explained by the decay of the ground state $a_{1}(1260)$ into $K^{*} \bar{K}$ and subsequent rescattering through a triangle singularity into the observed final state $f_{0}(980) \pi$ without the need of a new genuine $a_{1}$ resonance. The effect of the triangle singularity, which is expected to be present, is sufficient to explain the observation.

We gratefully acknowledge the support of the CERN management and staff as well as the skills and efforts of the technicians of our collaborating institutions. We would like to thank Bastian Kubis for useful discussions on the scalar form factor. This work was made possible by the financial support of our funding agencies: MEYS Grant No. LG13031 (Czech Republic); FP7 HadronPhysics3 Grant No. 283286 (European Union); CEA, P2I, and P.-J. L. was supported by ANR (France) with P2IO LabEx (ANR-10-LBX-0038) in the framework "Investissements d'Avenir" (ANR-11-IDEX-003-01); BMBF Collaborative Research Project 05P2018COMPASS, W. D. and M.F. were supported by the DFG Cluster of Excellence Origin and Structure of the Universe" ([52]), M. G. was supported by the DFG Research Training Group Programmes 1102 and 2044 (Germany); B. Sen Fund (India); Academy of Sciences and Humanities (Israel); INFN (Italy); MEXT and JSPS, Grants No. 8002006, No. 20540299, and No. 18540281, Daiko and Yamada Foundations (Japan); NCN Grant No. 2017/26/M/ST2/00498 (Poland); FCT Grants
No. CERN/FIS-PAR/0007/2017 and No. CERN/FIS-PAR/ 0022/2019 (Portugal); CERN-RFBR Grant No. 12-02-91500, Presidential Grant No. NSh-999.2014.2 (Russia); MST (Taiwan); and NSF (U.S.).

\footnotetext{
${ }^{\mathrm{a}}$ Deceased

b Also at: Department of Physics, Pusan National University, Busan 609-735, Republic of Korea and Physics Department, Brookhaven National Laboratory, Upton, New York 11973, USA.

${ }^{c}$ Also at: Abdus Salam ICTP, 34151 Trieste, Italy.

${ }^{\mathrm{d}}$ Also at: Chubu University, Kasugai, Aichi 487-8501, Japan.

eAlso at: Department of Physics, National Central University, 300 Jhongda Road, Jhongli 32001, Taiwan.

${ }^{\mathrm{f}}$ Also at: KEK, 1-1 Oho, Tsukuba, Ibaraki 305-0801, Japan. ${ }^{g}$ Present address: Physikalisches Institut, Universität Bonn, 53115 Bonn, Germany.

${ }^{\mathrm{h}}$ Also at: Moscow Institute of Physics and Technology, Moscow Region 141700, Russia.

${ }^{\mathrm{i}}$ Also at: Yerevan Physics Institute, Alikhanian Br. Street, Yerevan, Armenia, 0036.

${ }^{\mathrm{j}}$ Also at: Department of Physics, National Kaohsiung Normal University, Kaohsiung County 824, Taiwan.

${ }^{k}$ Also at: Institut für Theoretische Physik, Universität Tübingen, 72076 Tübingen, Germany.

${ }^{\mathrm{l}}$ Present address: Brookhaven National Laboratory, Upton, New York 11973, USA.

${ }^{\mathrm{m}}$ Also at: University of Eastern Piedmont, 15100 Alessandria, Italy.

${ }^{\mathrm{n}}$ Retired from Ludwig-Maximilian-Universität, München, Germany.

${ }^{\circ}$ Present address: III. Physikalisches Institut, RWTH Aachen University, 52056 Aachen, Germany.

${ }^{\mathrm{p}}$ Present address: Universität Hamburg, 20146 Hamburg, Germany.
}

[1] M. Tanabashi et al. (Particle Data Group), Phys. Rev. D 98, 030001 (2018).

[2] D. Ebert, R. N. Faustov, and V. O. Galkin, Phys. Rev. D 79, 114029 (2009).

[3] E. Eichten, K. Gottfried, T. Kinoshita, K. D. Lane, and T.-M. Yan, Phys. Rev. D 17, 3090 (1978); 21, 313(E) (1980).

[4] B. Ketzer, Proc. Sci., QNP2012 (2012) 025.

[5] C. A. Meyer and E. S. Swanson, Prog. Part. Nucl. Phys. 82, $21(2015)$.

[6] A. Esposito, A. Pilloni, and A. D. Polosa, Phys. Rep. 668, 1 (2017).

[7] F.-K. Guo, C. Hanhart, Ulf-G. Meißner, Q. Wang, Q. Zhao, and B.-S. Zou, Rev. Mod. Phys. 90, 015004 (2018).

[8] S. L. Olsen, T. Skwarnicki, and D. Zieminska, Rev. Mod. Phys. 90, 015003 (2018).

[9] A. Rodas et al. (JPAC Collaboration), Phys. Rev. Lett. 122, 042002 (2019).

[10] J. J. Dudek, R. G. Edwards, P. Guo, and C. E. Thomas (Hadron Spectrum Collaboration), Phys. Rev. D 88, 094505 (2013).

[11] P. Abbon et al. (COMPASS Collaboration), Nucl. Instrum. Methods Phys. Res., Sect. A 577, 455 (2007). 
[12] P. Abbon et al. (COMPASS Collaboration), Nucl. Instrum. Methods Phys. Res., Sect. A 779, 69 (2015).

[13] B. Ketzer, B. Grube, and D. Ryabchikov, Prog. Part. Nucl. Phys. 113, 103755 (2020).

[14] C. Adolph et al. (COMPASS Collaboration), Phys. Rev. D 95, 032004 (2017).

[15] C. Adolph et al. (COMPASS Collaboration), Phys. Rev. Lett. 115, 082001 (2015).

[16] F. Krinner, D. Greenwald, D. Ryabchikov, B. Grube, and S. Paul, Phys. Rev. D 97, 114008 (2018).

[17] K. Chen, C.-Q. Pang, X. Liu, and T. Matsuki, Phys. Rev. D 91, 074025 (2015).

[18] A. V. Anisovich, V. V. Anisovich, and A. V. Sarantsev, Phys. Rev. D 62, 051502(R) (2000).

[19] M. Mikhasenko, B. Ketzer, and A. Sarantsev, Phys. Rev. D 91, 094015 (2015).

[20] F. Aceti, L. R. Dai, and E. Oset, Phys. Rev. D 94, 096015 (2016).

[21] H.-X. Chen, E.-L. Cui, W. Chen, T. G. Steele, X. Liu, and S.-L. Zhu, Phys. Rev. D 91, 094022 (2015).

[22] T. Gutsche, M. A. Ivanov, J. G. Körner, V. E. Lyubovitskij, and K. Xu, Phys. Rev. D 96, 114004 (2017).

[23] J.-L. Basdevant and E. L. Berger, Phys. Rev. Lett. 114, 192001 (2015).

[24] V. N. Gribov, Y. L. Dokshitzer, and J. Nyiri, Strong Interactions of Hadrons at High Energies: Gribov Lectures on Theoretical Physics (Cambridge University Press, Cambridge, England, 2009).

[25] R. Eden, Rep. Prog. Phys. 34, 995 (1971).

[26] F.-K. Guo, X.-H. Liu, and S. Sakai, Prog. Part. Nucl. Phys. 112, 103757 (2020).

[27] J.-L. Basdevant and E. L. Berger, Phys. Rev. D 16, 657 (1977).

[28] M. Mikhasenko, Y. Wunderlich, A. Jackura, V. Mathieu, A. Pilloni, B. Ketzer, and A. P. Szczepaniak, J. High Energy Phys. 08 (2019) 080.

[29] A. P. Szczepaniak, Phys. Lett. B 747, 410 (2015).

[30] S. X. Nakamura and K. Tsushima, Phys. Rev. D 100, 051502(R) (2019).

[31] D. Herndon, P. Soding, and R. J. Cashmore, Phys. Rev. D 11, 3165 (1975).
[32] I. J. R. Aitchison, Phys. Rev. 137, B1070 (1965).

[33] N. N. Khuri and S. B. Treiman, Phys. Rev. 119, 1115 (1960).

[34] R. Pasquier and J. Y. Pasquier, Phys. Rev. 170, 1294 (1968).

[35] R. Pasquier and J. Y. Pasquier, Phys. Rev. 177, 2482 (1969).

[36] I. J. R. Aitchison and J. J. Brehm, Phys. Lett. 84B, 349 (1979).

[37] F. Niecknig and B. Kubis, J. High Energy Phys. 10 (2015) 142.

[38] F. Niecknig, B. Kubis, and S. P. Schneider, Eur. Phys. J. C 72, 2014 (2012).

[39] I. V. Danilkin, C. Fernandez-Ramirez, P. Guo, V. Mathieu, D. Schott, M. Shi, and A. P. Szczepaniak, Phys. Rev. D 91, 094029 (2015).

[40] C. Schmid, Phys. Rev. 154, 1363 (1967).

[41] A. P. Szczepaniak, Phys. Lett. B 757, 61 (2016).

[42] L. Landau, Nucl. Phys. 13, 181 (1959).

[43] See Supplemental Material at http://link.aps.org/ supplemental/10.1103/PhysRevLett.127.082501, which includes Refs. [44-47], for the extended description of possible triangle diagrams, a summary of systematic studies, details on the calculation, and a set of figures of the fit results all for $11 t^{\prime}$ bins.

[44] J. M. Blatt and V.F. Weisskopf, Theoretical Nuclear Physics (Wiley, New York, 1952).

[45] F. Von Hippel and C. Quigg, Phys. Rev. D 5, 624 (1972).

[46] J. B. Bronzan and C. Kacser, Phys. Rev. 132, 2703 (1963).

[47] M. Mikhasenko, A. Pilloni, A. Jackura, M. Albaladejo, C. Fernandez-Ramirez, V. Mathieu, J. Nys, A. Rodas, B. Ketzer, and A. P. Szczepaniak (JPAC Collaboration), Phys. Rev. D 98, 096021 (2018).

[48] See data tables in HEPData repository, http://www.hepdata .net/record/82958.

[49] G. Ascoli, L. M. Jones, B. Weinstein, and H. W. Wyld, Phys. Rev. D 8, 3894 (1973).

[50] M. Aghasyan et al. (COMPASS Collaboration), Phys. Rev. D 98, 092003 (2018).

[51] B. Efron and R. Tibshirani, Stat. Sci. 1, 54 (1986).

[52] https://www.universe-cluster.de/. 